\title{
Luigi Zingone
}

Università degli Studi e Campus (Novedrate, Włochy) e-mail: luigizingone@yahoo.it

ORCID 0000-0002-4993-4328

DOI: $10.15290 /$ mhi.2019.18.02.02

\section{Il Genius Loci nell'era della globalizzazione}

\author{
Abstract \\ Genius Loci in the era of globalization
}

In a globalized society a single identity is no longer verifiable, as happened in the Middle Ages, but as many identities as the cultural and social forms that can be found in communities.

From the considerations above reported, it would seem not only to have produced a profound rupture between past and present, but also that the meaning of the Genius Loci can be relegated to a historical and not an actual concept.

In the writer's opinion, the Genius Loci simply experiments with yet another change not very dissimilar to the many that we have been witnessing from the Roman era to the more recent one. This research claims to establish if:

i) it is possible to enhance localization, meant as the spirit of the place, through internationalization;

ii) there is a relationship between the spirit of the Genius Loci and the corporate strategies of internationalization in the era of globalization.

Key words: Genius Loci, globalization, internationalization, globalized society

\section{Introduzione}

Nella modernità, riferirsi al Genius Loci significa identificare gli aspetti caratteristici e/o distintivi di un luogo. Secondo Daniela Poli con il termine luogo 
ci si riferisce ad "un insieme identitario dotato di confini certi" ${ }^{1}$ ". Lidia Decandia sostiene che: "Il luogo è territorio e paesaggio. Territorio e paesaggio sottoposti a dinamiche identitarie, in cui sono presenti i termini di stabilità e continuità, che si manifestano secondo i caratteri di unitarietà e differenza ${ }^{2 "}$.

Definire, dunque, un luogo comporta ineluttabilmente una riflessione sull'identità dei popoli nonché sulla morfologia del territorio, con la conseguenza, tra le altre, della riconoscibilità che sovente corrisponde all'unicità del luogo medesimo.

Negli ultimi decenni, tuttavia, le società moderne hanno generato un allentamento dei legami con il luogo. La globalizzazione e l'aumento progressivo e significativo della mobilità hanno trasformato i luoghi, dapprima caratterizzati da stanzialità, in luoghi di transito nei quali non è possibile stabilire un rapporto abitativo in senso tradizionale.

In una società globalizzata non è più riscontrabile, così come accadeva nel medioevo, una identità univoca, ma tante identità quante sono le forme culturali e sociali che in essa è possibile ritrovare.

Dalle considerazioni appena svolte parrebbe non solo essersi prodotta una profonda rottura tra passato e presente ma sembrerebbe che il significato del Genius Loci possa essere relegato ad un concetto storico e non attuale.

Invero, a giudizio dello scrivente, il Genius Loci sperimenta semplicemente l'ennesimo cambiamento non molto dissimile dai tanti ai quali si è assistito dall'epoca romana a quella più recente.

La presente ricerca ha la pretesa di stabilire se:

i) sia possibile valorizzare la localizzazione, intesa come lo spirito del luogo, attraverso l'internazionalizzazione;

ii) esista una relazione tra lo spirito del Genius Loci con le strategie aziendali di internazionalizzazione nell'epoca della globalizzazione.

1 D. Poli, Il cartografo-biografo come attore della rappresentazione dello spazio in comune, in P. Castelnovi, Il senso del paesaggio, IRES, Torino, p. 205.

2 L. Decandia, Dell' identità. Saggio sui luoghi: per una critica della razionalità urbanistica, Rubbettino, Soveria Mannelli 2000, p.206. L'autrice analizza le condizioni storiche di apparizione del luogo affinché si possa parlare di identità del luogo, occorrono pratiche di appropriazione e significazione dello spazio (delimitazione che lo demarchi dal circostante - in tempi antichi dal caos -, mediante pratiche specifiche, di ordine rituale, simbolico, toponomastico, ordinativo, ecc.; la costituzione di un centro, di gerarchie e interrelazioni funzionali e simboliche); un sapere del governo del luogo basato sulla sua specifica configurazione, e dunque la necessità di tramandarne le regole e le pratiche; forme di memoria attiva e selettiva che garantiscano un dinamismo coerente con l'identità; statuti locali e regole consuetudinarie che assicurino la costruzione comunitaria dello spazio locale nella sua singolarità. 


\section{Capitolo 1. Il Genius Loci}

Nella mitologia romana il Genius Loci rappresentava lo spirito protettore di un luogo e si identificava con la figura di un serpente.

Secondo Servio, nullus locus sine Genio ${ }^{3}$. Il Genius Loci rappresentava, dunque, il genio del luogo abitato e frequentato dall'uomo.

Nell'attualità il significato del Genius Loci non ha perso di significatività, ma anzi si è evoluto a tal punto da essere identificabile e, pertanto, declinato in molte discipline umanistiche e scientifiche.

Con il tempo il significato si è modificato ed ha assunto un carattere trasversale.

In architettura il termine è adottato per individuare un approccio fenomenologico allo studio dell'ambiente, all'interazione tra luogo fisico e identità, al significato culturale e quello emozionale che viene attribuito a un luogo, a uno spazio o a una città.

Il Genius Loci sopravvive alle strutture funzionali in continua evoluzione e conferisce un carattere indelebile alle città e al paesaggio, attraverso diversi fenomeni urbani, tutti parte di una esperienza unica e riconoscibile.

Il critico Achille Bonito Oliva sostenne ${ }^{4}$ che il Genius Loci rappresentasse la ricerca dell'identità e il recupero della soggettività nell'arte.

Enzo Battara ${ }^{5}$ definisce la "glocalizzazione" non solo come la localizzazione della globalizzazione ma come il tentativo di recuperare il concetto di "genio" capace di dare anima alla materia. Secondo l'autore bisogna andare oltre la manualità, investendo anche sulle nuove tecnologie, senza mai dimenticare però la propria origine. Il linguaggio artistico deve essere quanto più contemporaneo possibile senza per questo perdere le radici.

\section{Capitolo 2. \\ Internazionalizzazione, analisi dell'attrattività e dell'accessibilità e Genius Loci}

\subsection{L'analisi preliminare nel processo di internazionalizzazione}

I mutamenti intervenuti negli ultimi decenni nel contesto competitivo in cui nascono, operano e si sviluppano le aziende, hanno sovvertito le strategie di sviluppo delle stesse nonché le modalità di gestione della concorrenza.

Le aziende che operano in un luogo preciso, non possono non interessarsi ai comportamenti adottati dalle organizzazioni facenti parte di altri sistemi

3 Nessun luogo è senza un Genio.

4 Intervista rilasciata dell'autore nell'anno 2004.

5 E. Battara, ne l'articolo dal titolo "Genius gloci: l'arte del territorio". 
nazionali; ciò in quanto nell'attuale contesto competitivo la distanza non può più essere considerata una protezione contro la concorrenza. Ne consegue, pertanto, che i processi competitivi si svolgono in un contesto sovranazionale.

L'integrazione dei mercati ha accentuato la tensione concorrenziale nella maggior parte dei settori economici.

Essendosi moltiplicate le possibilità di raffronto fra beni/servizi così come l'offerta, le aziende si trovano a dover far fronte a nuove minacce e a doversi confrontare con nuovi rivali, con rinnovate e sempre più sofisticate regole di competizione, con consumatori sempre più consapevoli ed esigenti.

Le aziende orientate ad adottare una strategia di internazionalizzazione devono preliminarmente valutare l'attrattività e l'accessibilità dei paesi verso i quali indirizzare la propria offerta.

L'analisi dell'attrattività presuppone, tra gli altri, l'esame delle caratteristiche e le dimensioni della domanda, i fattori critici di successo, il grado di compatibilità dei prodotti.

Le aziende, per individuare i paesi verso i quali indirizzare il processo di internazionalizzazione, devono tener conto anche del grado di accessibilità mediante l'apprezzamento dell'ambiente competitivo, dei canali di comunicazione, dei canali di distribuzione ${ }^{6}$.

Le barriere artificiali, da qualificare come "manipolazioni amministrative" dei flussi di beni, fattori produttivi e informazioni, attuate dai governi nazionali mediante scelte di politica economica, rappresentano un ulteriore elemento che incide nell'analisi dell'accessibilità.

Malgrado l'impegno profuso dall'Organizzazione Mondiale del Commercio (OMC o WTO) finalizzato alla riduzione delle barriere artificiali, non esiste alcuno Stato nel quale gli scambi con l'estero siano completamente liberi e sottratti a ogni controllo ${ }^{7}$.

\subsection{Genius loci quale fattore strategico per le imprese locali}

Il contesto economico sociale mondiale, durante gli ultimi decenni, ha subito, ad una velocità superiore rispetto a quella caratterizzante epoche passate, molteplici cambiamenti.

La globalizzazione ha assunto un ruolo di assoluto protagonismo nel citato scenario internazionale imponendosi quale elemento generatore di modificazione delle regole macroeconomiche, delle strategie aziendali, delle abitudini dei consumatori, delle caratteristiche sociali dei popoli.

$6 \quad$ L. Zingone, F. Ruiz Moreno, Estrategias y modalidades de ingreso para competir en mercato internacionales. Publicaciones Universidad de Alicante, 2014.

7 E. Valdani, G. Bertoli, Mercati internazionali e marketing. Egea, Milano 2010. 
Le norme che regolavano i mercati hanno subito modificazioni profonde dovute alla disgregazione dei confini abituali. Nel passato la competizione, nella maggior parte dei settori, si caratterizzava dalla presenza di un numero di concorrenti limitato e noto.

Nell'attualità, anche a seguito della rivoluzione digitale, pur in assenza della pianificazione di una strategia finalizzata all'internazionalizzazione, è astrattamente possibile che ogni azienda possa collocare la propria offerta oltre i confini del mercato fisico di appartenenza.

La crisi che ha colpito nel 2008 le maggiori economie mondiali, peraltro, ha determinato un ridimensionamento della domanda interna ${ }^{8}$, rendendo necessario, e in taluni casi indispensabile per la sopravvivenza stessa delle imprese, dirigere l'offerta verso un mercato divenuto ineluttabilmente unico. Il predetto processo è stato accompagnato dalla diffusione dei nuovi strumenti informatici e da internet. Le imprese di medio - grandi dimensioni hanno reagito attraverso la predisposizione di un piano di internazionalizzazione prevedendo, in taluni casi, anche la presenza fisica di stabilimenti e/o uffici nei paesi stranieri. Attraverso investimenti generalmente di modesto valore anche le imprese di piccole dimensioni e le microimprese hanno potuto partecipare al processo di cambiamento de quo, avviando le vendite on-line.

La rete ha rappresentato e rappresenta una modalità per divulgare le caratteristiche dei prodotti tipici di un luogo e, di conseguenza, uno strumento ulteriore per dare impulso alle vendite anche nei mercati internazionali.

In definitiva, la globalizzazione e la rivoluzione digitale non hanno rappresentato dei fattori negativi per le imprese produttrici di beni legati al contesto territoriale nel quale sono allocate: al contrario, hanno dato impulso all'offerta, determinando un effetto domino positivo attraverso il sostentamento e l'arricchimento del luogo.

\subsection{Genius Loci e internazionalizzazione: dalla coesistenza alla conversione in fattore strategico}

Nei processi di internazionalizzazione meno strutturati, l'identificazione dei Paesi esteri verso i quali dirigere l'offerta dell'azienda avveniva attraverso l'individuazione dei mercati più prossimi rispetto al Paese d'origine. Generalmente, minore è la distanza esistente tra due Paesi, minore è il rischio percepito dall'azienda. Per questo ragione, sovente la scelta dei Paesi risulta circoscritta a quelli limitrofi, poiché la prossimità geografica comporta di solito maggiore similarità culturale, migliore conoscenza del mercato estero e un più agevole accesso alle informazioni.

8 https://www.difesa.it/InformazioniDellaDifesa/periodico/.../gambini-pignatti.pdf. 
L'offerta dell'azienda, tuttavia, grazie all'incessante sviluppo delle vie di comunicazione, della tecnologia e delle strategie di supply chain, patisce sempre meno le distanze geografiche. I fattori che richiedono una sempre maggiore e più sofisticata comprensione sono rappresentati dai gusti, dalle preferenze e dalla cultura dei consumatori che vivono nel contesto sociale dove l'azienda intende dirigere la predetta offerta.

Nel corso degli anni i mercati hanno sperimentato plurimi casi di prodotti di successo nei confini nazionali che si sono rivelati fallimenti di successo in taluni mercati internazionali.

Agli inizi degli anni '70, ad esempio, la multinazionale svizzera Nestlè ha cercato di imporre in Africa il proprio latte in polvere per l'alimentazione dei neonati.

La strategia di internazionalizzazione, pur essendo stata oggetto di pianificazione e supportata da un'azione di marketing imponente, si è rivelata del tutto inadeguata a causa di una erronea analisi dell'ambiente generale del mercato target. La multinazionale, difatti, non aveva tenuto in debita considerazione che l'acqua potabile (elemento, come noto, essenziale per la trasformazione del latte in polvere in latte), in molti Paesi africani è scarsamente disponibile oppure, sovente, contaminata.

Il detto tentativo di penetrazione aggressiva del mercato si è rivelato fallimentare sotto l'aspetto commerciale ed ha prodotto effetti negativi sulla reputazione della marca. L'azienda, difatti, fu accusata di uccidere più bambini con i suoi sostituti del latte di quanti ne avrebbe salvati dalla scarsa nutrizione. La Mattel, azienda produttrice della famosa bambola Barbie, ha penetrato il mercato cinese realizzando un grande store a Shanghai. Nonostante una strategia prudente basata nell'ingresso in una città storicamente aperta al commercio internazionale (a differenza delle altre realtà urbane cinesi), i prodotti della Mattel non hanno riscosso il successo sperato. Le ragioni del fallimento sono state originate da una sottovalutazione delle caratteristiche socioculturali del Paese target: i cinesi, infatti, attribuiscono ai giocattoli un ruolo educativo; avendo valutato come frivoli i prodotti offerti dalla multinazionale (in particolare la famosa bambola Barbie), si sono astenuti dall'acquisto.

Dal punto di vista aziendale una delle reazioni al superamento dei fallimenti nei mercati internazionali è avvenuta attraverso i metodi del marketing differenziato e della segmentazione, che concepiscono il mercato come un'entità eterogenea, formata da tanti segmenti tra loro omogenei. Porter ${ }^{9}$ definisce il processo di segmentazione come quello per cui si scompone un mercato in singole unità (target) che vengono poi riagglomerate, sulla base di variabili, in gruppi sociali ed economici più o meno ampi, definiti segmenti.

9 Michael E. Porter, Il Vantaggio Competitivo, Einaudi, 2011. 
Le variabili attraverso le quali avviene la segmentazione si possono distinguere in: i) geografiche ${ }^{10}$; ii) sociodemografiche ${ }^{11}$; iii) psicografiche ${ }^{12}$; iv) comportamentali ${ }^{13}$.

Il marketing differenziato parte dall'assunto che esistano tanti gruppi di potenziali clienti, a ciascuno dei quali si devono proporre più prodotti o servizi diversificati. All'esito della segmentazione vengono identificate le caratteristiche socioculturali di un luogo, di un mercato, e, di conseguenza, viene praticato un adattamento dell'offerta ai bisogni dei singoli segmenti.

Rispetto alla strategia di marketing indifferenziato (caratterizzata da un'offerta standard), con il marketing differenziato la gamma dei prodotti/servizi offerti è più ricca e postula ampie possibilità di personalizzazione.

Le tecniche di segmentazione che si sono sviluppate nel corso degli ultimi decenni, basate fondamentalmente sulla raccolta di dati statistici, hanno certamente contribuito ad una maggiore conoscenza dei consumatori e, dunque, dei mercati.

La definizione dei mercati obiettivo e la loro attrattività, tuttavia, non può essere misurata, come succedeva nel passato anche recente, unicamente attraverso l'applicazione della matrice di Ansoff ${ }^{14}$ e del modello delle 5 forze di Porter ${ }^{15}$.

Gli sforzi profusi dalle aziende nella raccolta dei dati, quelli prodotti da società specializzate anche attraverso il web e con l'impiego di sempre più sofisticati algoritmi, tuttavia, hanno sovente scontato il limite di prescindere da un articolato studio sociologico capace di interpretare e non manipolare il Genius Loci del mercato target.

L'evoluzione del concetto di Genius Loci nel tempo e la conseguente trasversalità che lo connota attualmente permettono di identificarlo, tra l'altro, con il carattere e l'insieme delle peculiarità socioculturali propri di una comunità, di un popolo. Partendo dalla predetta declinazione, appare allora essenziale per ogni azienda prendere in considerazione il Genius Loci quale fattore competitivo indispensabile nella predisposizione e nella esecuzione di una strategia di internazionalizzazione.

10 La predetta variabile si caratterizza per l'identificazione dell'area geografica, della popolazione e del clima.

11 Si procede ad una segmentazione per età, sesso, reddito, occupazione, titolo di studio, livello di istruzione, numero di componenti in famiglia e religione.

12 La segmentazione avviene per classe sociale, stile di vita e personalità.

13 La segmentazione avviene secondo: i) i vantaggi ricercati dal consumatore rispetto al prodotto; ii) la consapevolezza circa le caratteristiche ed i servizi ante e post-vendita; iii) la fedeltà alla marca.

14 Antonio Ferrandina, Il marketing strategico per le PMI. Gli strumenti per elaborare piani e strategie vincenti. Franco Angeli edizione 2012.

15 Michael E. Porter, op. cit. 


\subsection{Genius Loci Program}

Il Genius Loci Program è stato ideato ${ }^{16}$ allo scopo di sviluppare un programma di ricerca capace di offrire:

i) alle aziende locali un contributo alle riflessioni sempre più complesse sul tema della globalizzazione;

ii) alle multinazionali ed alle imprese interessate a penetrare i mercati internazionali gli strumenti di analisi capaci di meglio interpretare il sub ambiente socioculturale che caratterizza un dato luogo.

Con il detto programma di ricerca si intendono chiarire e misurare le potenzialità locali che un'azienda o un'istituzione può vantare nel mercato globale. Partendo da una selezione dei talenti locali (Genius Loci) e delle qualità uniche espresse dai valori culturali di un luogo, il programma si propone, tra gli altri, di selezionare e valorizzare quegli elementi che potranno essere apprezzati nel mondo globale, in sintonia con le tendenze in altri paesi o continenti.

Parimenti, le imprese interessate a penetrare i mercati internazionali, attraverso fonti di informazione secondaria (fornite proprio dal programma), hanno la possibilità di conoscere le caratteristiche sociali, i valori culturali, i gusti e le preferenze di un popolo.

Attraverso il contributo di corrispondenti presenti in circa 40 città distribuite in 25 Paesi $^{17}$, il Genius Loci Program identifica gli elementi caratterizzanti i luoghi offrendo spunti strategici per la gestione dei mercati sempre più coinvolti nella dialettica fra Unico e Universale.

\section{Capitolo 3. \\ Genius Loci nell'era digitale}

\subsection{Il Genius Loci Digitale nella sua declinazione storico-artistica}

L'avvento della rivoluzione digitale ha reso possibile, tra gli altri, la creazione e la diffusione di sistemi di rappresentazione e comunicazione del tutto innovativi.

Attraverso la comparsa di una serie di nuovi strumenti di programmazione per oggetti multimediali, è stato possibile rendere sempre più integrabili tra loro grafica animata e interattiva, video, oggetti grafici tridimensionali, fotografia panoramica immersiva, archivi iconografici e testuali, strutture ipertestuali. La predetta integrazione ha favorito la nascita dell'ipermedialità.

L'uso di dette tecnologie ha permesso, tra gli altri, la promozione del patrimonio culturale e identitario delle comunità attraverso una indefinita pluralità

16 Ideato dalla Future Concept Lab.

17 I Paesi analizzati nel Genius Loci Program sono Australia, Belgio, Brasile, Cile, Cina, Colombia, Corea, Finlandia, Francia, Germania, Giappone, Grecia, India, Islanda, Israele, Italia, Olanda, Regno Unito, Russia, Spagna, Stati Uniti, Sudafrica, Svezia, Thailandia. 
di informazioni che vengono veicolate sui media digitali. La conoscenza ed il "senso" del luogo si acquisiscono grazie ad una combinazione innovativa di materiali multimediali prodotti ad hoc, capaci di riprodurre sul web delle vere e proprie visite guidate ${ }^{18}$. Tuttavia, il compimento di quello che può essere definito Genius Loci Digitale necessita non solo di immagini ma, bensì, della rappresentazione anche testuale e delle interrelazioni intercorrenti tra fatti storici, schemi urbanistici, opere d'arte, architettura, simboli civili e religiosi.

\subsection{Il Genius Loci Digitale nella sua declinazione economico-sociale}

Con l'avvento della rivoluzione digitale il ruolo del consumatore ha subito delle profonde modificazioni. Mentre prima assumeva un ruolo quasi passivo delegando alle imprese il compito di indirizzare stimolare e condizionare le opzioni di acquisto, oggi il consumatore ha assunto un ruolo proattivo (anche grazie ad una diversa consapevolezza acquisita attraverso la rete, la quale ha consentito una drastica riduzione delle asimmetrie informative).

Il "prosumer"19 antepone al processo di acquisto una fase finalizzata all'acquisizione di informazioni, condivide esperienze, definisce la personalizzazione del bene e/o del servizio. La rete garantisce e facilita l'accesso alle informazioni e consente di condividere molteplici contenuti; di conseguenza, ogni persona è in grado di interagire e generare un cambiamento di opinioni. Le plurime opinioni divulgate nella rete, dunque, influenzano le scelte di acquisto degli altri consumatori. In definitiva, il consumatore è esposto a molti stimoli, ma quelli che si sedimentano sono quelli forniti da chi sa generare costantemente partecipazione e discussioni, fornendo argomenti a valore aggiunto e che, in prima persona, volontariamente e non per conto terzi si erge a portavoce del brand ${ }^{20}$. Individuato un bisogno, un desiderio del consumatore, l'azienda impiega le risorse possibili allo scopo di ideare, progettare o migliorare un prodotto/servizio funzionale al bisogno captato (in altri termini, dopo la fase dell'ascolto del mercato l'azienda perviene alla risposta). Con l'avvento della rivoluzione digitale il percorso classico citato è cambiato al punto che domanda ed offerta hanno trovato delle nuove modalità d'interazione.

18 Davide Gasperi, Strategie di spazializzazione dei contenuti nel GeniusLoci Digitale, "Storicamente", 9 (2013), no. 24.

19 Alvin Toffler nell'opera intitolata The Third Wave, del 1980, coniò il termine "prosumer" quando predisse che il ruolo di produttore e consumatore avrebbe cominciato a fondersi e confondersi (sebbene ne parli già nel libro Future Shock dal 1970). Toffler immaginò un mercato fortemente saturo dal momento in cui la produzione di massa di merci standardizzate cominciava a soddisfare domande basiche dei consumatori. Per continuare l'incremento dei profitti, le aziende avrebbero avviato un processo di personalizzazione di massa, cioè la produzione massiva di prodotti altamente personalizzati. Tuttavia, per raggiungere un alto livello di personalizzazione era necessario che i consumatori prendessero parte al processo soprattutto nel definire le caratteristiche estetiche progettuali dei prodotti. 
La fase di determinazione ${ }^{21}$ del bisogno/desiderio latente del consumatore e la fase d' ideazione della migliore risposta (nuovo prodotto o miglioria di quello esistente) non sono più ben distinte, ma sempre più spesso avviene la loro concisione per merito delle nuove modalità di relazione e interazione. Le nuove opportunità di business, l'ideazione e la progettazione dei nuovi prodotti, nascono dal dialogo dell'azienda con le community, i clienti e i consumatori.

È la domanda (sempre più rappresentata dai membri delle community) che idea, crea, apporta migliorie ${ }^{22}$ e giudica un prodotto.

Il diffondersi degli acquisti on line di beni e/o servizi non deve indurre a pensare che questa modalità sia stata il risultato dell'applicazione di logiche di acquisto orientate al price cutting (inteso come decurtazione); piuttosto, essa è stata la come conseguenza di cambiamenti che hanno interessato:

i) il tempo di prenotazione o di acquisto del prodotto ${ }^{23}$;

ii) il luogo in cui avviene l'acquisto ${ }^{24}$.

In definitiva, rispetto al passato, le community dell'era digitale rappresentano una evoluzione del popolo che tradizionalmente occupava un luogo ben definito i cui confini attuali sono essi stessi privi di significato. Si viene a formare, dunque, un'unione priva di confini ma che identifica comunque un gruppo di persone che si uniscono per condividere degli interessi, soddisfare dei bisogni. Le caratteristiche sociali della community, allora, identificano il Genius Loci.

\section{Conclusioni}

Le dottrine che si sono sviluppate nelle plurime discipline che si interessano al Genius Loci concordano nel sancirne una difficile coesistenza con la globalizzazione a tal punto da domandarsi se sia possibile, nell'epoca attuale, parlare ancora del genio del luogo. Ciò, tra l'altro, in quanto non sarebbe possibile individuare, come nel passato, i luoghi (attraverso dei confini definiti e certi di una data comunità), e di conseguenza, una identità univoca.

21 Nella fase iniziale volta ad intercettare i bisogni, si ricorre all'utilizzo di ricerche di tipo quali-quantitativo.

22 Un caso italiano di successo raggiunto attraverso l'interazione con gli utenti, è stato il lancio della nuova Fiat 500; la piattaforma 500wantsyou, ha raccolto i contributi e i suggerimenti di milioni di utenti.

23 Il fattore in questione è detto anche "dynamic pricing", ed è alla base della strategia adottata dalle compagnie aeree. A titolo di esempio si pensi alle politiche di pricing adottate dalla Ryanair, la quale varia il prezzo del biglietto aereo in base all'approssimarsi della data di partenza desiderata.

24 Il fattore spaziale caratterizza la strategia di tipo "multichannel pricing", nella quale il prezzo varia in base alla diversificazione dell'offerta (canale online/offline); ad esempio da Mediaword acquistando un elettrodomestico online si gode del $9 \%$ di sconto rispetto a chi compra direttamente nel punto vendita. 
La globalizzazione avrebbe reso, dunque, il Genius Loci un concetto storicamente datato e non attuale.

Il concetto di Genius Loci, come noto, nel corso dei secoli, si è trasformato sino ad assumere un carattere trasversale.

La globalizzazione, invero, non ha sancito la fine del Genius Loci, ma ha rappresentato un elemento di rottura rispetto al passato, favorendone l'ennesimo mutamento.

Il processo di integrazione dei mercati, tra gli altri, attraverso la diffusione della mobilità ha reso difficile, per molti essere umani, stabilire un rapporto abitativo in senso tradizionale-stanziale, ed ha conferito alla società tante identità quante sono le forme culturali e sociali che in essa è possibile ritrovare.

In piena era digitale, attraverso la diffusione delle community, si sono formate unioni prive di confini ma che identificano un gruppo di persone che si uniscono per condividere degli interessi e soddisfare dei bisogni. Gli elementi di unione di una community favoriscono lo sviluppo di una identità e, pertanto, il Genius Loci.

\subsection{La valorizzazione della localizzazione attraverso l'internazionalizzazione}

Le imprese che operano in una qualunque area geografica non possono ignorare le strategie ed i comportamenti adottati dalle aziende facenti parte di altri sistemi nazionali, perché nell'attuale contesto competitivo la distanza non è più una protezione contro la concorrenza. $\mathrm{Da}$ ciò ne consegue che i processi competitivi avvengono a livello sovranazionale.

La globalizzazione unitamente al diffondersi della rivoluzione digitale e al manifestarsi dell'ultima crisi economica mondiale, hanno spinto le imprese (a prescindere dalle dimensioni) a rivolgere la propria offerta oltre i confini fisici del proprio mercato per dirigersi verso il mercato unico.

Dunque, mentre in passato l'internazionalizzazione era di esclusivo dominio delle multinazionali, ora anche le imprese di piccole dimensioni e le microimprese partecipano a questo processo attraverso il sistema delle vendite on-line.

La rete ha permesso di creare una vetrina informativa, accessibile a livello globale, capace di valorizzare il genio del luogo attraverso la vendita dei prodotti tipici.

\subsection{Il Genius Loci quale valore aggiunto nel processo di internazionalizzazione}

Uno degli elementi di successo alla base di un processo di internazionalizzazione è rappresentato dalla adeguata pianificazione delle strategie di marketing differenziato che tengano conto delle caratteristiche complessive del mercato target. 
Nel passato le politiche di marketing ritenute di successo erano orientare ad incidere, fino a modificare, le abitudini, i gusti, le preferenze, il carattere, l'identità di un gruppo.

La rivoluzione digitale, avendo reso il consumatore più attento e consapevole, ha indotto le aziende a modificare le politiche finalizzate alla manipolazione, obbligandole a stabilire una nuova relazione improntata alla comprensione ed al rispetto del Genius Loci.

La conoscenza del Genius Loci che identifica un mercato e/o una community rappresenta, dunque, non solo un fattore vitale capace di decretare il successo ovvero l'insuccesso dell'offerta diretta ai mercati internazionali, ma può costituire il valore aggiunto della strategia di internazionalizzazione.

\section{Bibliografia}

Decandia L., Dell'identità. Saggio sui luoghi: per una critica della razionalità urbanistica, Rubbettino, Soveria Mannelli 2000.

Ferrandina A., Il marketing strategico per le PMI. Gli strumenti per elaborare piani e strategie vincenti. Franco Angeli edizione 2012.

Gasperi D., Strategie di spazializzazione dei contenuti nel GeniusLoci Digitale, "Storicamente", 9 (2013), no. 24.

Poli D., Il cartografo-biografo come attore della rappresentazione dello spazio in comune, in P. Castelnovi, Il senso del paesaggio, IRES, Torino.

Porter M.E., Il Vantaggio Competitivo, Einaudi, 2011.

Toffler A., The Third Wave, WorldofBooks (Goring-By-Sea, WS, Regno Unito), 1980.

Valdani E., Bertoli G., Mercati internazionali e marketing. Egea, Milano 2010.

Zingone L., Ruiz Moreno F., Estrategias y modalidades de ingreso para competir en mercato internacionales. Publicaciones Universidad de Alicante, 2014.

\section{SUMMARY}

\section{Genius Loci in the era of globalization}

In Roman mythology the Genius Loci represented the protective spirit of a place and identified with the figure of a snake. The Genius Loci therefore represented the genius of the place inhabited and frequented by man. The meaning of the Genius Loci has not lost significance, but rather has evolved to the point of being identifiable. Over time the meaning has changed and has taken on a transversal character.

Globalization and the progressive and significant increase in mobility have transformed places, initially characterized by permanence, into transit places where it is not possible to establish a housing relationship in the traditional sense. 
In the present, even following the digital revolution, even in the absence of the planning of a strategy aimed at internationalization, it is abstractly possible that each company can place its offer beyond the borders of the physical market to which it belongs. The crisis that hit the major world economies in 2008, however, led to a reduction in domestic demand, making it necessary, and in some cases indispensable for the survival of companies, to direct the offer towards a market that has become inevitably unique. The aforementioned process was accompanied by the diffusion of the new IT tools and the Internet. Ultimately, globalization and the digital revolution did not represent negative factors for companies producing goods related to the territorial context in which they are allocated: on the contrary, they boosted the offer, resulting in a positive domino effect through livelihood and enrichment of the place.

Compared to the past, the communities of the digital age represent an evolution of the people who traditionally occupied a well-defined place whose current borders are themselves meaningless. Therefore, a union without boundaries is formed, but which nevertheless identifies a group of people who come together to share interests, satisfy needs. The social characteristics of the community, then, identify the Genius Loci. 
